\title{
Tumor size as a predictor for prognosis of patients with surgical IIIA-N2 non-small cell lung cancer after surgery
}

\author{
Kunpeng Zhang", Jiahao Cai", Chunlai Lu, Qiaoliang Zhu, Cheng Zhan, Yaxing Shen, Jie Gu, Di Ge \\ Department of Thoracic Surgery, Zhongshan Hospital, Fudan University, Shanghai, China \\ Contributions: (I) Conception and design: D Ge; (II) Administrative support: J Gu, D Ge; (III) Provision of study materials or patients: K Zhang, J \\ Cai; (IV) Collection and assembly of data: C Lu, C Zhan, Y Shen; (V) Data analysis and interpretation: K Zhang, J Cai; (VI) Manuscript writing: All \\ authors; (VII) Final approval of manuscript: All authors. \\ \#These authors contributed equally to this work. \\ Correspondence to: Di Ge, MD; Jie Gu, MD. Department of Thoracic Surgery, Zhongshan Hospital, Fudan University, 180 Fenglin Road, Xuhui \\ District, Shanghai 200032, China. Email: ge.di@zs-hospital.sh.cn; gu.jie3@zs-hospital.sh.cn.
}

Background: The $8^{\text {th }}$ edition of the American Joint Committee on Cancer staging system for lung cancer made major revisions to T staging, especially the size division of stage II/III patients. However, the value of tumor size in the postoperative prognosis of IIIA-N2 non-small cell lung cancer (NSCLC) is seldom mentioned, and survival data of such patients should be re-evaluated according to the $8^{\text {th }}$ edition staging system.

Methods: Patients with IIIA-N2 NSCLC after surgery were identified in the Surveillance, Epidemiology, and End Results database $(n=4,128)$. All patients were stratified according to tumor size, 5 -year overall survival (OS) was then compared. Cox regression analysis was used to determine the value of size to discriminate patients with prognostic differences and establish a predictive nomogram system. Patients with IIIA-N2 NSCLC from our own institute $(\mathrm{n}=583)$ were used to validate the results.

Results: The prognosis of patients with tumor sizes of $0-2,2-4$ and 4-5 cm differed greatly from each other in the training cohort, with 5 -year OS rates of $53.7 \%, 43.9 \%$ and $36.9 \%$ respectively $(\mathrm{P}<0.001)$, in the validation cohort, the rates were $54.1 \%, 38.4 \%$ and $33.8 \%$ respectively. Tumor size $>2 \mathrm{~cm}$ was considered an independent risk factor compared to the $\leq 2 \mathrm{~cm}$ group in the Cox regression analysis: $2-4 \mathrm{~cm}(\mathrm{HR}=1.25$, 1.12-1.39; $\mathrm{P}<0.001), 4-5 \mathrm{~cm}(\mathrm{HR}=1.51,1.32-1.39 ; \mathrm{P}<0.001)$, the validation cohort showed the same trend. The concordance index of the training set was $0.634(0.622-0.646)$, while that of the validation set was 0.716 (0.686-0.746). The calibration plot showed optimal consistency between the nomogram predicted survival and observed survival.

Conclusions: Tumors with different sizes showed significant postoperative survival differences among patients with IIIA-N2 NSCLC. Tumor size should be considered when making surgery decisions in such patients, with tumor size $\leq 2 \mathrm{~cm}$ showing considerably better prognosis.

Keywords: Stage IIIA; N2; surgery; size; nomogram

Submitted Mar 12, 2021. Accepted for publication May 27, 2021.

doi: $10.21037 /$ jtd-21-428

View this article at: https://dx.doi.org/10.21037/jtd-21-428

\section{Introduction}

Despite advances in targeted therapy and immunotherapy, the long-term survival of patients with advanced-stage lung cancer remains poor (1), and a large proportion of patients have mediastinal lymph node (LN) metastases when they are first diagnosed. Moreover, whether surgery should be used to treat pathologically confirmed stage IIIA (N2) non-small cell lung cancer (NSCLC) patients remains controversial (2), randomized controlled trials have failed to confirm that surgery as part of multidisciplinary treatment 
has any advantage over radical chemoradiotherapy in pathologically confirmed IIIA-N2 patients $(3,4)$. However, previous studies have included patients with T3-T4 tumors according to the $7^{\text {th }}$ edition of the American Joint Committee on Cancer (AJCC) staging system, and IIIA (N2) NSCLC is a heterogeneous diagnosis. Surgery combined with multidisciplinary therapy is widely accepted and is recommended by the National Comprehensive Cancer Network (NCCN) to treat selected patients with N2 NSCLC, namely those T1-2 or non-invasive T3 NSCLC (5). Furthermore, $\mathrm{N}$ status is still the most important factor in the development of therapeutic strategies; in particular, surgery is not recommended in patients with multi-station $\mathrm{N} 2$ and bulky N2, because such patients have relatively poor prognosis (6). However T stage also plays an important role in the postoperative survival of patients with N2 NSCLC $(7,8)$, and tumor size is the greatest contributor to $\mathrm{T}$ stage among patients with $\mathrm{T} 1-2$ or non-invasive T3 NSCLC.

The $8^{\text {th }}$ edition of the AJCC lung cancer TNM staging system made considerable revisions to the size division of $\mathrm{T}$ stage classification, stage IIIA-N2 was confined within T1-2 at a tumor size of $\leq 5 \mathrm{~cm}$; smaller than in the $7^{\text {th }}$ edition staging system; this indicated the importance of tumor size in such patients (9). Tumor size was reported to be correlated with postoperative outcomes in patients with IIIA-N2 NSCLC (7). However, no systemic assessment of survival differences according to tumor size has been conducted in these patients; therefore, it remains unclear whether and how clinicians should consider size when making decisions about surgery. Hence, we aimed to evaluate survival differences in patients with different sizes of resected stage IIIA-N2 NSCLC; patients were stratified into 0-2, 2-4 and 4-5 cm groups, based on their survival differences. A nomogram was established to predict the survival of patients after surgery and was validated with independent data.

We present the following article in accordance with the TRIPOD reporting checklist (available at https://dx.doi. org/10.21037/jtd-21-428).

\section{Methods}

\section{Patient population}

The SEER data were acquired from a public access database using SEER*Stat. We only extracted patients who underwent surgery and were histologically diagnosed with
NSCLC as their first primary malignant tumor between 2005 and 2015. Patients were restaged according to the $8^{\text {th }}$ edition staging system using records in the Collaborative Stage Data Collection System; pathological information after surgery such as tumor size, invasion extension, LN metastasis, and distant metastasis were obtained from the Collaborative Stage Data. Chemotherapy and radiotherapy treatment information was also acquired from the "radiation/chemotherapy databases" of SEER, but only the sequence of radiotherapy with surgery was recorded in the database; the sequence of chemotherapy with surgery was not available. Moreover, chemotherapy regimens were not available for a more detailed analysis. Only surgical IIIA-N2 patients were selected, and patients with no clear data on tumor size, surgery type, or positive LNs were excluded.

To match the time span of the SEER data, patients who underwent surgery between 2005 and 2015 in the thoracic surgery department of Shanghai Zhongshan Hospital, Fudan University, were extracted and restaged according to the $8^{\text {th }}$ edition staging system using postoperative pathological information. Clinical characteristics and restaging were based on the hospital's clinical records, with no survival data available before the study was completed. Follow-up of patients was performed through telephone every 3 months until September 2018. Patients pathologically diagnosed with IIIA-N2 NSCLC were included in the validation cohort. All patients provided informed consent for the use of their clinical information before surgery and the study was approved by the institutional review board. Patients from both SEER and Zhongshan with pathological stage IIB-N1 NSCLC were also extracted as a baseline for overall survival (OS) comparison. The study was conducted in accordance with the Declaration of Helsinki (as revised in 2013).

\section{Statistical analyses}

The $\chi^{2}$ test was used to compare categorical variables, and analysis of variance to compare continuous variables. OS and survival curves were estimated using the Kaplan-Meier method and Cox proportional hazards model analysis. A Cox regression model was established with SEER data to obtain the hazard ratio (HR) of each tumor size compared with tumors of $\leq 1 \mathrm{~cm}$. Regression models were assessed using the concordance index (C-index) with " $\mathrm{R}$ " software. A nomogram was depicted with " $\mathrm{R}$ " based on the Cox regression model. SPSS and " $R$ " software were used for data analysis. 


\section{Results}

\section{Patients and characteristics}

Of 5,512 patients with stage IIIA-N2 NSCLC, 1,384 had incomplete information about grade, surgery method, examined LNs, positive LNs or tumor sizes; hence, all these patients were excluded, 4,128 patients with complete information from SEER were included. A total of 5,412 patients with pathological stage IIB-N1 NSCLC were extracted for baseline comparison at the same time. A total of 583 patients with stage IIIA-N2 NSCLC treated at Zhongshan Hospital, Shanghai, China were included; 25 of them had no follow-up information, and the median follow-up time was 43 months. A baseline comparison was performed in 374 patients with pathological stage IIB-N1 NSCLC. The characteristics of both cohorts are listed in Tables 1 and 2. The mean age of the SEER cohort was 65.1 years, $32.3 \%$ of them were over 70 years old, while the mean age of the validation cohort was 58.8 years, and $11.3 \%$ of them were over 70 years. Lobectomy was the major surgery type in both cohorts; the SEER cohort had a higher rate of local resection (10.5\% vs. $1.0 \%)$. Adenocarcinoma accounted for the largest proportion of tumors $(73.7 \%$ in the SEER and $80.9 \%$ in the validation cohort). Fewer patients in the validation cohort received chemotherapy (42.2\% vs. $71.2 \%$ ). In the SEER cohort, $7.8 \%$ of patients received pre-operative radiotherapy, and $34.8 \%$ received post-operative radiotherapy, while the equivalent rates were $1.4 \%$ and $8.2 \%$ in the validation cohort. The 5 -year survival rates were $45.5 \%$ and $40.9 \%$ in the SEER and validation cohorts, with median survival times of 51 and 48 months, respectively.

\section{Prognosis was worse in patients with greater tumor size}

The Kaplan-Meier survival curves of patients with different size hierarchies are shown in Figure 1. The 5-year survival rates of patients were $65.8 \%, 52.3 \%, 44.4 \%, 43.4 \%$ and $36.9 \%$ in the 1-, 2-, 3-, 4- and 5-cm hierarchy groups respectively. Cox regression analysis was conducted, adjusted for age, sex, surgery type, grade, size hierarchy, examined LNs, positive LNs, central bronchus invasion, pleural invasion, and chemotherapy. The HR of patients with different tumor size hierarchies compared to those with $1 \mathrm{~cm}$ tumors is shown in Figure $2 A$. The OS curves of different size hierarchies, ascertained with Cox regression analysis, showed similar trends to the Kaplan-Meier survival curves (Figure 2B). Based on these survival differences, patients were stratified into $0-2,2-4$ and $4-5 \mathrm{~cm}$ groups in both cohorts. The 5-year survival rate was significantly lower when the tumor size was greater in the SEER cohort (53.7\%, 43.9\%, 36.9\%, $\mathrm{P}<0.001$ ), and the median survival times were 68, 48, and 36 months, respectively (Figure $3 A$ ); the IIB-N1 group had a similar 5 -year OS to the $0-2 \mathrm{~cm}$ group $(55.3 \% ; \mathrm{P}=0.126)$, as well as a median survival time of 77 months. In the validation cohort, the 5 -year OS rates of the three size groups were $54.1 \%, 38.4 \%$ and $33.8 \%$, with median survival times of 64,46 , and 40 months, respectively (Figure 3B). The $0-2 \mathrm{~cm}$ group had better survival than the 2-4 cm $(\mathrm{P}=0.008)$ and $4-5 \mathrm{~cm}$ groups $(\mathrm{P}=0.005)$, but similar survival to the IIB-N1 NSCLC group from the validation cohort $(\mathrm{P}=0.77)$; the $2-4 \mathrm{~cm}$ group showed no significant differences from the $4-5 \mathrm{~cm}$ group $(\mathrm{P}=0.417)$. Cox regression analysis showed the same trend as the KaplanMeier method in both cohorts (data not shown).

\section{Tumor size was correlated with other risk factors}

The characteristics of patients in different size groups from the SEER and the validation cohorts are listed in Tables 1 and 2. Tumor size was correlated with other risk factors, in the SEER cohort the large tumor group tended to have more patients aged $>70$ years $(\mathrm{P}=0.002)$, more male patients $(\mathrm{P}<0.001)$, more grade III/IV patients $(\mathrm{P}=0.008)$, more patients with $\geq 5$ positive $L N s(\mathrm{P}<0.001)$, more pleural or central bronchus invasion $(\mathrm{P}<0.001)$, more patients with $\geq 6$ positive LNs $(\mathrm{P}<0.001)$, and more instances of pre-operative radiotherapy $(\mathrm{P}=0.006)$. However, the large tumor group had fewer patients with adenocarcinoma $(\mathrm{P}<0.001)$ and less local resection $(\mathrm{P}<0.001)$ than the $0-2 \mathrm{~cm}$ tumor group. In the validation cohort, larger tumor size was correlated with more males $(\mathrm{P}=0.002)$, more central bronchus invasion $(\mathrm{P}<0.001)$ and less adenocarcinoma $(\mathrm{P}<0.001)$.

\section{Developing a prognostic nomogram}

A Cox regression model was established based on10-year survival data, including all the correlated risk factors. Age $>70$ years, male sex, local resection, grade III/IV, tumor size of $2-4$ or $4-5 \mathrm{~cm}, \leq 5$ examined LNs, $\geq 4$ positive LNs, central bronchus invasion, pleural invasion, and absence of chemotherapy were all considered independent risk factors for unfavorable survival. The $\mathrm{P}$ values of all risk factors with univariate and multivariate analyses and HRs are listed in Table 3. The cut-off values of age, examined LNs count, and positive LNs count were determined using the Cox 
Table 1 Characteristics of pathological IIIA-N2 patients receiving surgery stratified by tumor size from SEER

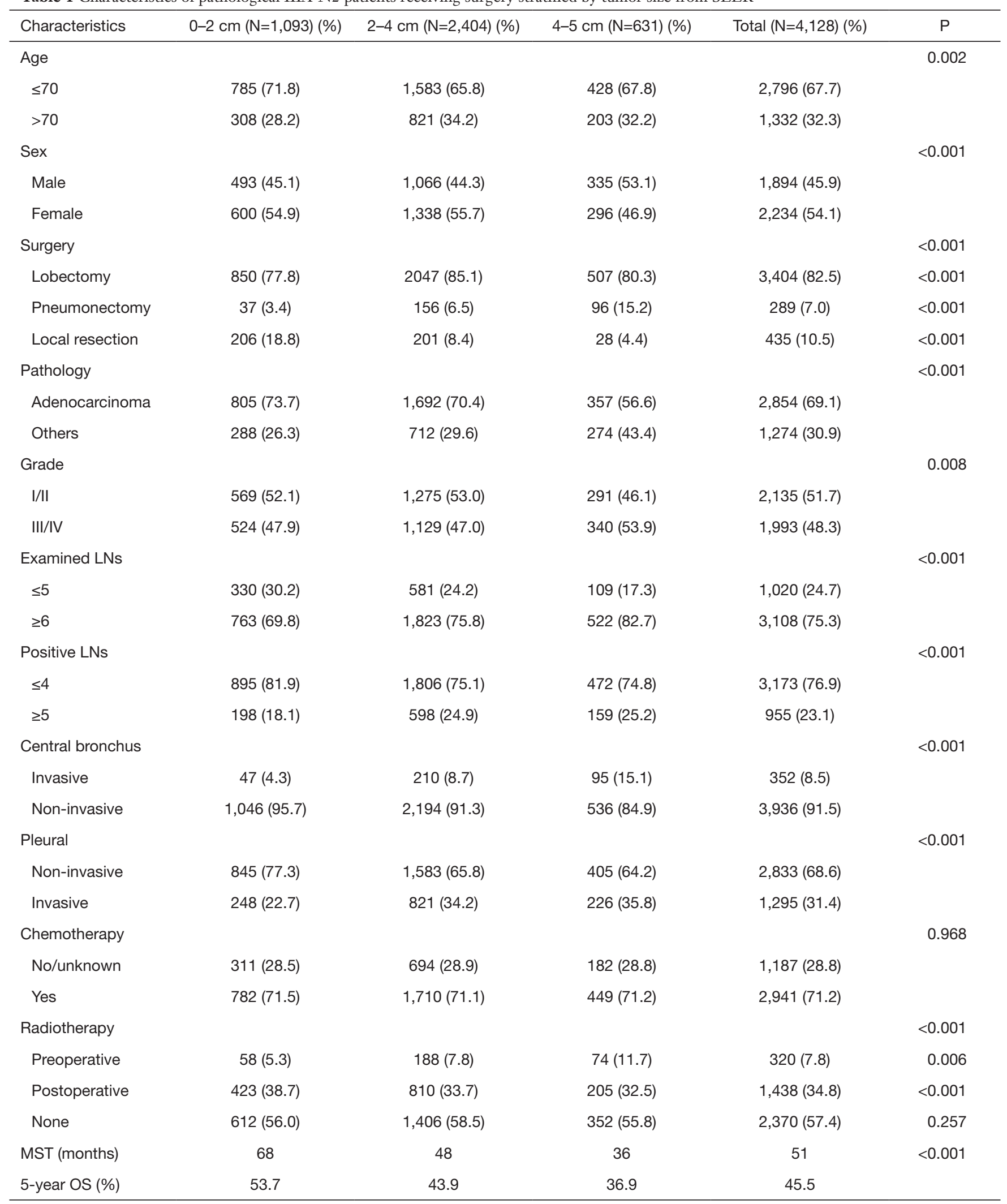

LN, lymph node. 
Table 2 Characteristics of pathological IIIA-N2 patients receiving surgery stratified by tumor size from the validation cohort

\begin{tabular}{|c|c|c|c|c|c|}
\hline Characteristics & $0-2 \mathrm{~cm}(\mathrm{~N}=115)$ & $2-4 \mathrm{~cm}(\mathrm{~N}=382)$ & $4-5 \mathrm{~cm}(\mathrm{~N}=86)$ & Total $(\mathrm{N}=583)$ & $P$ \\
\hline$\leq 70$ & $104(90.4)$ & $335(87.7)$ & $78(90.7)$ & $517(88.7)$ & \\
\hline$>70$ & $11(9.6)$ & 47 (12.3) & $8(9.3)$ & $66(11.3)$ & \\
\hline Sex & & & & & 0.002 \\
\hline Female & $36(31.3)$ & 165 (43.2) & $22(25.6)$ & 223 (38.3) & \\
\hline Surgery & & & & & 0.47 \\
\hline Lobectomy & $112(97.4)$ & $363(95.0)$ & 81 (94.2) & $556(95.4)$ & 0.487 \\
\hline Pneumonectomy & $2(1.7)$ & $14(3.7)$ & $5(5.8)$ & $21(3.6)$ & 0.306 \\
\hline Others & $22(19.1)$ & $106(27.7)$ & $49(57.0)$ & $177(30.4)$ & \\
\hline Grade & & & & & 0.958 \\
\hline$I / I I$ & $45(39.1)$ & $149(39.0)$ & $35(40.7)$ & 229 (39.3) & \\
\hline III/IV & $70(60.9)$ & $233(61.0)$ & $51(59.3)$ & $354(60.7)$ & \\
\hline Examined LNs & & & & & 0.098 \\
\hline$\leq 5$ & $6(5.2)$ & $7(1.8)$ & $4(4.7)$ & $17(2.9)$ & \\
\hline$\geq 6$ & 109 (94.8) & 375 (98.2) & 82 (95.3) & $566(97.1)$ & \\
\hline Non-invasive & 103 (89.6) & 296 (77.5) & $51(59.3)$ & 450 (77.2) & \\
\hline Pleural & & & & & 0.186 \\
\hline Non-invasive & $53(46.1)$ & $177(46.3)$ & $49(57.0)$ & 279 (47.9) & \\
\hline Invasive & $62(53.9)$ & $205(53.7)$ & $37(43.0)$ & $304(52.1)$ & \\
\hline Chemotherapy & & & & & 0.445 \\
\hline No/unknown & $62(53.9)$ & $228(59.7)$ & $47(54.7)$ & $337(57.8)$ & \\
\hline Yes & $53(46.1)$ & $154(40.3)$ & 39 (45.3) & 246 (42.2) & \\
\hline Radiotherapy & & & & & 0.757 \\
\hline Preoperative & $3(2.6)$ & $4(1.0)$ & $1(1.2)$ & $8(1.4)$ & 0.444 \\
\hline Postoperative & $10(8.7)$ & $30(7.9)$ & $8(9.3)$ & $48(8.2)$ & 0.889 \\
\hline None & $102(88.7)$ & $348(91.1)$ & 77 (89.5) & 527 (90.4) & 0.714 \\
\hline MST (months) & 64 & 46 & 40 & 48 & 0.012 \\
\hline 5-year OS (\%) & 54.1 & 38.4 & 33.8 & 40.9 & \\
\hline
\end{tabular}

LN, lymph node. 
regression model; age divided by 70 , examined LNs counts divided by 5 , and positive LNs count divided by 4 could achieve the best consistency and largest log-rank score. The C-index of the optimal Cox regression model was 0.634 (95\% CI: 0.622-0.646) in the training cohort, and the C-index of the model without size included was 0.628 (95\% CI: 0.616-0.640). The Cox regression model was validated with an independent validation cohort, and the $\mathrm{C}$-index was 0.716 (95\% CI: $0.686-0.746$ ) in the validation cohort; the C-index of the model without size included was $0.712(95 \%$

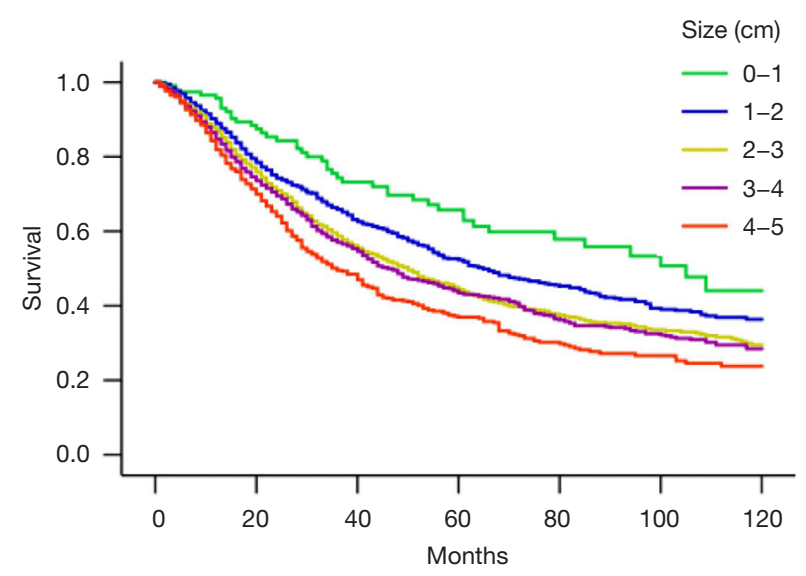

Figure 1 Kaplan-Meier curves of different tumor size hierarchy groups of postoperative patients with pathological IIIA-N2 nonsmall cell lung cancer in the SEER cohort.

A

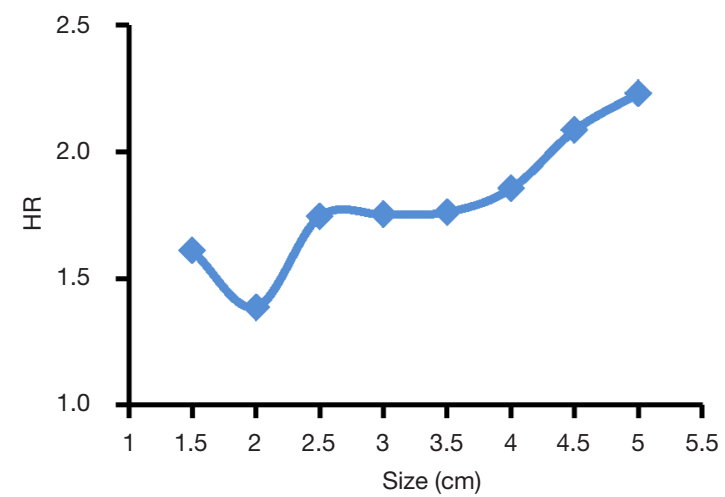

$\begin{array}{lllllllll}\text { Size }(\mathrm{cm}) & 1.5 & 2 & 2.5 & 3 & 3.5 & 4 & 4.5 & 5\end{array}$

$\begin{array}{lllllllll}\text { HR } & 1.61 & 1.39 & 1.74 & 1.75 & 1.76 & 1.85 & 2.08 & 2.23\end{array}$
CI: 0.682-0.742). A nomogram was devised with correlated variables included to estimate the probability of OS in the training cohort (Figure 4). The 5- and 10-year survival rates were estimated according to the total points of the patient. A calibration plot of the model presented an optimal prediction of 5-year OS in both cohorts compared with the actual observations (Figure 5).

\section{Conclusions}

The TNM staging system of the $8^{\text {th }}$ edition AJCC, demonstrated better survival stratification of $\mathrm{T}$ staging than the $7^{\text {th }}$ edition $(10,11)$. Stage N2 disease was separated into subgroups of stage IIIA and IIIB depending on whether T1-2 or T3-4 was present. In addition, a strict tumor size of $5 \mathrm{~cm}$ was implemented in T2, whereas the $7^{\text {th }}$ stipulated a tumor size of $7 \mathrm{~cm}$ (9). Stage IIIA shows better survival than IIIB after surgery, as confirmed in our previous studies, which found 5 -year OSs of $45.5 \%$ and $36.7 \%$, respectively, in the SEER. Therefore, we focused on stage IIIA-N2 NSCLC, which has a more promising prognosis, to assess the risk factors related to post-operative prognosis.

Mediastinal LN status was still the most important factor when devising treatment strategy in patients with IIIA-N2 NSCLC, and surgery was considered more frequently among skip (N2a1), single-station (N2a2), and non-bulky N2 patients to ensure better survival $(2,12,13)$. Positive LN counts, examined LN counts, and positive ratio of

B

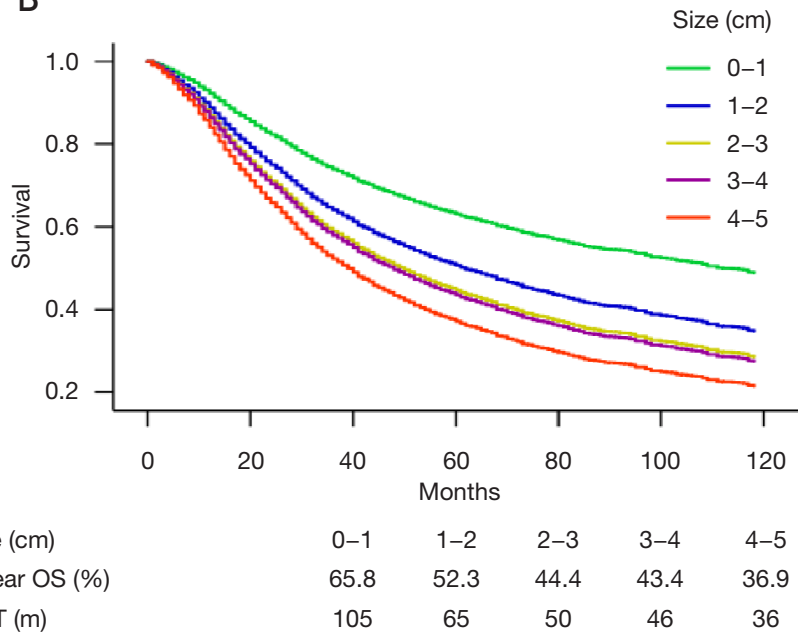

Figure 2 Hazard ratio and survival differences of different tumor size hierarchy groups in the SEER cohort. (A) Hazard ratio of pathological IIIA-N2 NSCLC patients with different tumor size groups compared to tumor size $\leq 1 \mathrm{~cm}$; (B) Cox regression analysis of survival differences between different tumor size groups. NSCLC, non-small cell lung cancer. 


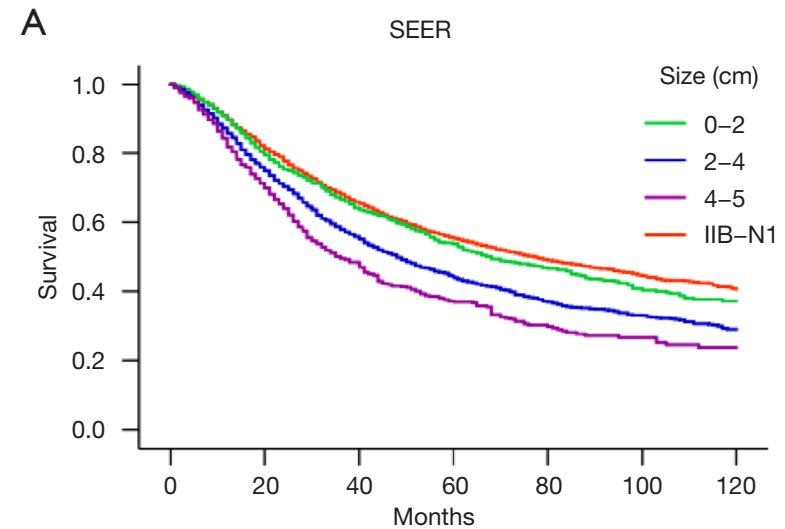

$\begin{array}{lcccc}\text { Size }(\mathrm{cm}) & \text { IIB-N1 } & 0-2 & 2-4 & 4-5 \\ \text { 5-year OS (\%) } & 55.3 & 53.7 & 43.9 & 36.9 \\ \text { MST (m) } & 77.0 & 68 & 48 & 36\end{array}$

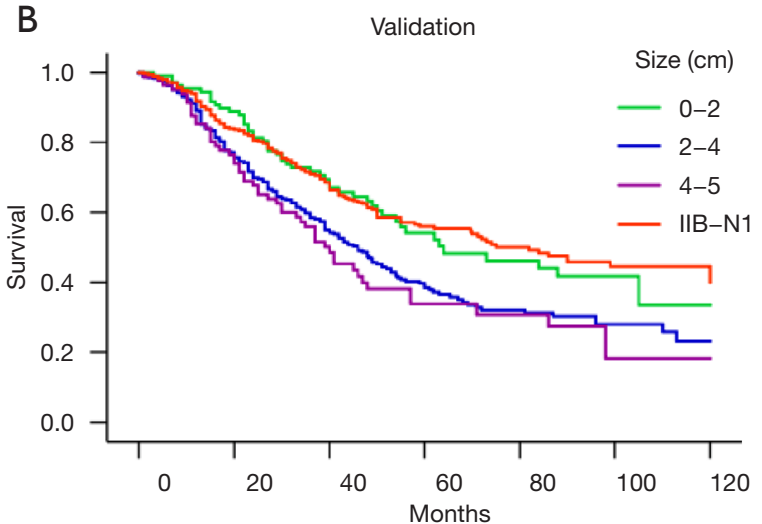

$\begin{array}{lcccc}\text { Size }(\mathrm{cm}) & \text { IIB-N1 } & 0-2 & 2-4 & 4-5 \\ \text { 5-year OS (\%) } & 56.0 & 54.1 & 38.4 & 33.8 \\ \text { MST (m) } & 75 & 64 & 46 & 40\end{array}$

Figure 3 Survival differences of patients stratified into 0-2, 2-4 and 4-5 cm tumor size groups. (A) Kaplan-Meier curves of IIIA-N2 nonsmall cell lung cancer patients stratified according to tumor size in the SEER cohort; (B) Kaplan-Meier curves of patients stratified according to tumor size in the validation cohort; Red line represents the IIB-N1 group in each cohort.

Table 3 Univariate and multivariate analysis of risk factors associated with 5-year overall survival of IIIA-N2 patients after surgery in SEER

\begin{tabular}{|c|c|c|c|}
\hline Variable & Univariate $(\mathrm{P})$ & \multicolumn{2}{|c|}{ Multivariate } \\
\hline Age (>70 vs. $\leq 70)$ & $<0.001$ & $1.38(1.26-1.51)$ & $<0.001$ \\
\hline Gender (male vs. female) & $<0.001$ & $1.32(1.21-1.44)$ & $<0.001$ \\
\hline \multicolumn{4}{|l|}{ Surgery } \\
\hline Local resection vs. lobectomy & $<0.001$ & $1.23(1.06-1.42)$ & 0.005 \\
\hline Pathology (adenocarcinoma vs. others) & 0.853 & - & 0.106 \\
\hline Grade (III/IV vs. I/II) & $<0.001$ & $1.15(1.05-1.25)$ & 0.002 \\
\hline Examined LNs ( $\geq 6$ vs. $\leq 5)$ & $<0.001$ & $1.41(1.27-1.56)$ & $<0.001$ \\
\hline Pleural (invasive vs. non-invasive) & $<0.001$ & $1.21(1.10-1.33)$ & $<0.001$ \\
\hline Tumor size & $<0.001$ & & \\
\hline $2-4$ vs. $0-2 \mathrm{~cm}$ & & $1.25(1.12-1.39)$ & $<0.001$ \\
\hline $4-5$ vs. $0-2 \mathrm{~cm}$ & & $1.51(1.32-1.73)$ & $<0.001$ \\
\hline Chemotherapy (no/unknown vs. yes) & $<0.001$ & $1.32(1.21-1.45)$ & $<0.001$ \\
\hline Radiotherapy (vs. no radiotherapy) & & & 0.109 \\
\hline Preoperative & 0.007 & - & 0.225 \\
\hline Postoperative & 0.018 & - & 0.189 \\
\hline
\end{tabular}

LN, lymph node. 


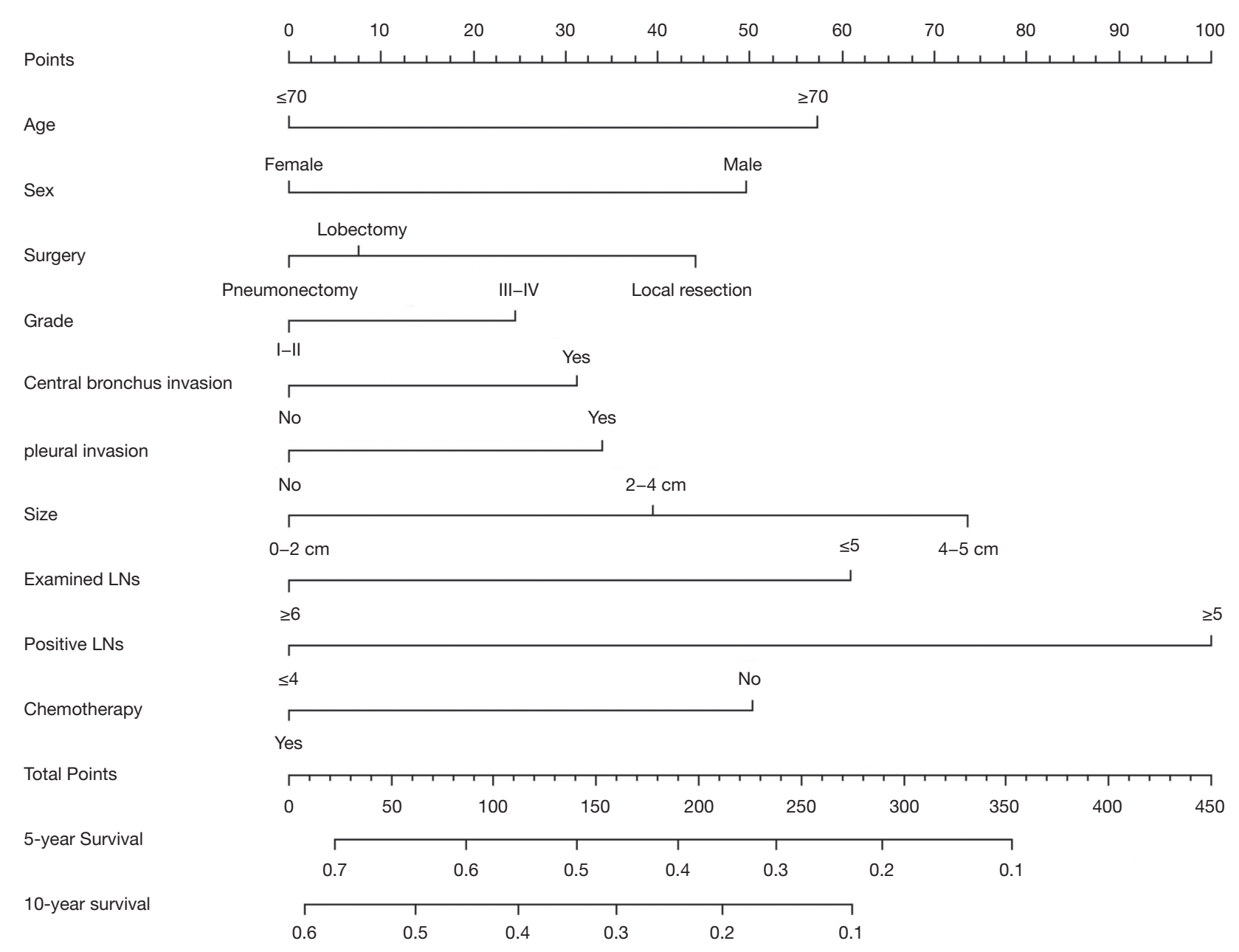

Figure 4 Nomogram of the Cox regression model predicting post-operative survival of patients with pathological N2 non-small cell lung cancer in the SEER cohort.

LNs are also correlated with the prognosis of N2 $(14,15)$. In the present study, we included examined LN counts and positive $\mathrm{LN}$ counts in the Cox regression analysis to eliminate the impact of $\mathrm{N}$ status. Positive $\mathrm{LN}$ counts $\geq 5$ represented worse $\mathrm{N}$ status, with unfavorable survival; as such, it was included and adjusted in the subsequent Cox regression analysis.

$\mathrm{T}$ stage information including tumor size and invasion extension, was also correlated with the prognosis of patients with IIIA-N2 NSCLC $(7,8,16)$. In the Cox regression analysis in a study by Mao et al., tumor size was included as a continuous variable related to postoperative survival in patients with IIIA-N2 NSCLC (8); that analysis based on the $7^{\text {th }}$ edition staging system. Chen et al. also reported that tumor measuring $1-2$ or $2-3 \mathrm{~cm}$ were risk factors compared to those measuring $0-1 \mathrm{~cm}$ among patients with $\mathrm{T} 1 \mathrm{~N} 2$
NSCLC (17). In a small sample of 77 cases, a tumor size of $<3 \mathrm{~cm}$ was proved a favorable prognostic factor among patients with IIIA-N2 NSCLC, according to the $8^{\text {th }}$ edition staging system (7). Additionally, in a whole population of surgical and non-surgical patients, $3 \mathrm{~cm}$ is the optimal cutoff point to separate T1 from T2 in the TNM staging system, but it is unknown whether $3 \mathrm{~cm}$ is the optimal cutoff point to discriminate postoperative survival differences in patients with IIIA-N2. Our results showed that patients with a tumor measuring $0-1 \mathrm{~cm}$ had the best survival, while the 1-2 $\mathrm{cm}$ hierarchy group had significantly better survival than the $2-3 \mathrm{~cm}$ group in both cohorts (Figure 1A). The $2-3 \mathrm{~cm}$ group had a 5 -year OS closer to the $3-4 \mathrm{~cm}$ group $(\mathrm{P}=0.452)$. We stratified the patients into three groups based on these survival differences; thus, the $0-1 \mathrm{~cm}$ hierarchy was stratified into the same group as the $1-2 \mathrm{~cm}$ hierarchy, 


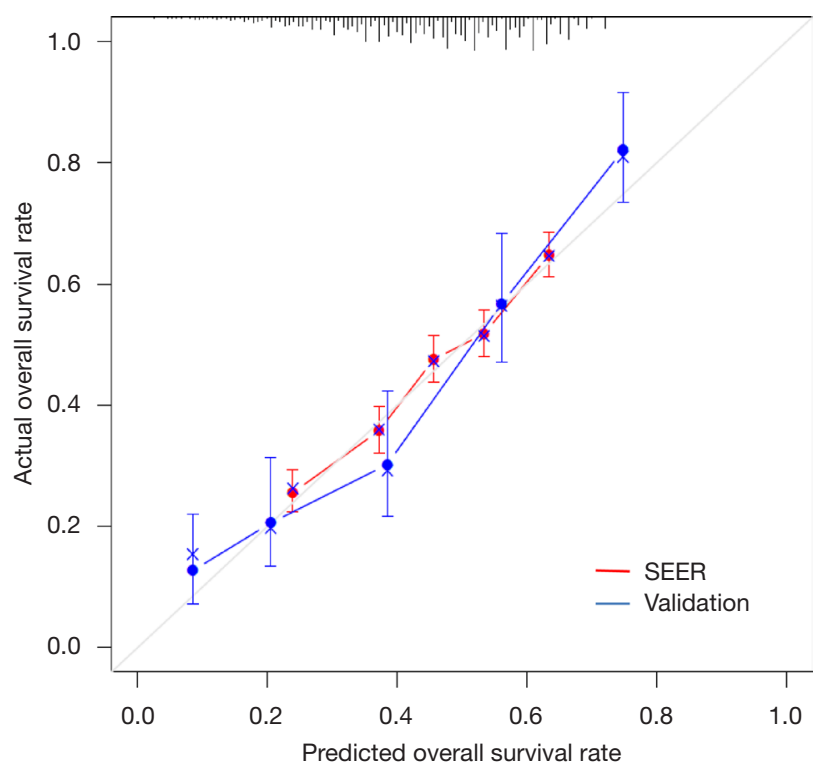

Figure 5 Calibration plot presenting the predicted 5-year overall survival compared to actual survival in the SEER (red) and validation cohorts (blue), as revealed by the Cox regression model.

while the $2-3 \mathrm{~cm}$ was stratified into the same group as the $3-4 \mathrm{~cm}$ hierarchy. These results suggest that $2 \mathrm{~cm}$ might be a better cut-off point to discriminate postoperative survival in patients with IIIA-N2 NSCLC.

The stratification of patients according to tumor size could better discriminate between survival differences. The 0-2 cm hierarchy group represented a low-risk group with significantly better OS than the larger size group $53.7 \%$ in the SEER and $54.1 \%$ in validation cohort). In fact, the OS in the $0-2 \mathrm{~cm}$ group was similar to that of the pathological IIB-N1 groups in both cohorts. The $4-5 \mathrm{~cm}$ group was a relative high-risk group with a worse 5 -year OS of $36.9 \%$ in the SEER cohort and $33.8 \%$ in the validation cohort. In the multivariate Cox regression analysis, stratified tumor size was also independent risk factor correlated with survival. The HR of the 4-5 $\mathrm{cm}$ group compared to the 0-2 cm was 1.51 (95\% CI: $1.32-1.73$ ), which was higher than the HR of all other risk factors except for positive LN count $\geq 5$ (1.75, 95\% CI: $1.58-1.94)$. This could also be seen on the nomogram, which indicated that tumor size was important in the prognosis of such patients. Larger tumor were correlated with many other risk factors, such as male sex, older age, more grade III/IV patients, more positive LNs, more central bronchus and pleural invasion. We speculated that larger tumors are also related to a higher rate of $\mathrm{R} 1$ resection and local recurrence rate, but we could not obtain enough original information to confirm this. However, some clues were the higher rate of central bronchus invasion and the pneumonectomy ratio, both of which indicated greater probability of difficult operations. All these factors contributed to the poor survival of patients with larger tumors. A nomogram with tumor size could better predict the prognosis of patients after surgery. The results suggested that tumor size should be carefully considered when making treatment decisions in patients with N2 NSCLC. One limitation of the present study was the lack of post-operative recurrence information and radical/palliative resection records, which may have allowed us to understand our results more.

Although our results showed that the $0-2 \mathrm{~cm}$ group had a good 5-year OS rate after surgery in both cohorts (53.7\% and $54.1 \%$ in the SEER and validation cohorts, respectively), mediastinoscopy to assess $\mathrm{LN}$ metastasis was still important for making treatment decisions. However, even though it is recommended in all patients with $\mathrm{N} 2$ NSCLC, mediastinoscopy is still far from widespread, and accurate multistation $\mathrm{N} 2$ assessment remains difficult before surgery. Therefore, tumor size is the most important factor to help clinicians make treatment decisions. We propose that if multiple $\mathrm{N} 2$ is not confirmed or multistation mediastinal $\mathrm{LN}$ assessment is unavailable, the $0-2 \mathrm{~cm}$ group should be strongly recommended for surgery. The median survival time of the $4-5 \mathrm{~cm}$ group (40 and 36 months) was far from that of the 0-2 cm group (68 and 64 months) and was only slightly better than the 29 months reported in 2018 among patients receiving definitive chemoradiation; in that study, neoadjuvant treatment followed by surgery was compared with definitive chemoradiation among patients with stage IIIA-N2 NSCLC (3). The time span was similar to that of the present study, but the patients were staged according to the $7^{\text {th }}$ edition TNM classification, which included more severe invasive tumors than our study. We speculate that the $4-5 \mathrm{~cm}$ group received limited added benefit from the surgery over the definitive chemoradiation treatment, and that the role of surgery in such patients should be assessed in randomized control trials.

Both adjuvant therapy and radical tumor or LN resection contributed to the prognosis after surgery in the present study. Although SEER data showed older age, higher rate of local resection, and fewer examined LNs, more patients received chemotherapy; meanwhile, the validation cohort had more central bronchus and pleural invasion, less chemotherapy treatment, but higher radical lobectomy rate and more examined LNs. As such, the 
survival data in each size hierarchy were similar. With the development of immunotherapy, definitive conformal radiation therapy (CRT) plus durvalumab proved superior to traditional CRT in a phase 3 PACIFIC study in patients with stage III NSCLC who showed no progression after chemoradiotherapy (18). The PACIFIC study achieved a median survival of 43.3 months among patients with $1-24 \%$ tumor cells expressing PD-L1, which was higher than the median survival time of patients with $\geq 25 \%$ PD-L1 expression. However, these results are still not comparable to the survival of $0-2 \mathrm{~cm}$ surgery group in the present study (68 and 64 months), and we believe that prognosis will be more promising if adjuvant or neoadjuvant durvalumab treatment were applied. Neoadjuvant immunotherapy with or without chemotherapy has proven effective in patients with resectable lung cancer, with a major pathological response in $40.5 \%$ to $57 \%$ of patients (19-21). We believe that resection of the primary lesion will benefit patients with N2 NSCLC who have shown better systemic treatment outcomes among well-selected candidates.

In conclusion, our results indicated that tumor size is important in the postoperative prognosis of patients with IIIA-N2 NSCLC; the 0-2 $\mathrm{cm}$ group showed better prognosis than any other groups, while the 4-5 cm group showed poor prognosis. A nomogram with tumor size included may better predict patient survival. Size should also be considered when developing treatment strategies for patients with N2 NSCLC awaiting surgery.

\section{Acknowledgments}

Funding: This work was supported by the National Nature Science Foundation of China [81872291], Department of Thoracic Surgery, Zhongshan Hospital, Fudan University.

\section{Footnote}

Reporting Checklist: The authors have completed the TRIPOD reporting checklist. Available at https://dx.doi. org/10.21037/jtd-21-428

Peer Review File: Available at https://dx.doi.org/10.21037/ jtd-21-428

Conflicts of Interest: All authors have completed the ICMJE uniform disclosure form (available at https://dx.doi. org/10.21037/jtd-21-428). The authors have no conflicts of interest to declare.
Ethical Statement: The authors are accountable for all aspects of the work in ensuring that questions related to the accuracy or integrity of any part of the work are appropriately investigated and resolved. The study was conducted in accordance with the Declaration of Helsinki (as revised in 2013). The study was approved by Ethics committee of Zhongshan Hospital, Fudan University (B2021-128).

Open Access Statement: This is an Open Access article distributed in accordance with the Creative Commons Attribution-NonCommercial-NoDerivs 4.0 International License (CC BY-NC-ND 4.0), which permits the noncommercial replication and distribution of the article with the strict proviso that no changes or edits are made and the original work is properly cited (including links to both the formal publication through the relevant DOI and the license). See: https://creativecommons.org/licenses/by-nc-nd/4.0/.

\section{References}

1. Siegel RL, Miller KD, Jemal A. Cancer statistics, 2019. CA Cancer J Clin 2019;69:7-34.

2. Tabchi S, Kassouf E, Rassy EE, et al. Management of stage III non-small cell lung cancer. Semin Oncol 2017;44:163-77.

3. Couñago F, Rodriguez de Dios N, Montemuino S, et al. Neoadjuvant treatment followed by surgery versus definitive chemoradiation in stage IIIA-N2 non-small cell lung cancer: A multi-institutional study by the oncologic group for the study of lung cancer (Spanish Radiation Oncology Society). Lung Cancer 2018;118:119-27.

4. Eberhardt WE, Pottgen C, Gauler TC, et al. Phase III Study of Surgery Versus Definitive Concurrent Chemoradiotherapy Boost in Patients With Resectable Stage IIIA(N2) and Selected IIIB Non-small cell Lung Cancer After Induction Chemotherapy and Concurrent Chemoradiotherapy (ESPATUE). J Clin Oncol 2015;33:4194-201.

5. Tan WL, Chua KLM, Lin CC, et al. Asian Thoracic Oncology Research Group expert consensus statement on optimal management of stage III non-small cell lung cancer. J Thorac Oncol 2020;15:324-43.

6. Zheng D, Wang Y, Li Y, et al. Predicting prognosis of post-chemotherapy patients with resected IIIA non-small cell lung cancer. J Thorac Dis 2018;10:4186-94.

7. Chen CY, Wu BR, Chen CH, et al. Prognostic value of tumor size in resected stage IIIA-N2 non-small cell lung 
cancer. J Clin Med 2020;9:1307.

8. Mao Q, Xia W, Dong G, et al. A nomogram to predict the survival of stage IIIA-N2 non-small cell lung cancer after surgery. J Thorac Cardiovasc Surg 2018;155:1784-1792.e3.

9. Detterbeck FC, Boffa DJ, Kim AW, et al. The Eighth Edition Lung Cancer Stage Classification. Chest 2017;151:193-203.

10. Sui $X$, Jiang $W$, Chen $H$, et al. Validation of the Stage Groupings in the Eighth Edition of the TNM Classification for Lung Cancer. J Thorac Oncol 2017;12:1679-86.

11. Koul R, Rathod S, Dubey A, et al. Comparison of 7th and 8th editions of the UICC/AJCC TNM staging for nonsmall cell lung cancer in a non-metastatic North American cohort undergoing primary radiation treatment. Lung Cancer 2018;123:116-20.

12. Postmus PE, Kerr KM, Oudkerk M, et al. Early and locally advanced non-small cell lung cancer (NSCLC): ESMO Clinical Practice Guidelines for diagnosis, treatment and follow-up. Ann Oncol 2017;28:iv1-iv21.

13. Wang L, Ye G, Xue L, et al. Skip N2 Metastasis in Pulmonary Adenocarcinoma: Good Prognosis Similar to N1 Disease. Clin Lung Cancer 2020;21:e423-e434.

14. Park JK, Moon Y. Prognosis of upstaged N1 and N2 disease after curative resection in patients with clinical N0 non-small cell lung cancer. J Thorac Dis 2019;11:1202-12.
15. Łochowski M, Łochowska B, Rębowski M, et al. Five-year survival analysis and prognostic factors in patients operated on for non-small cell lung cancer with N2 disease. J Thorac Dis 2018;10:3180-6.

16. Deng $\mathrm{W}, \mathrm{Xu} \mathrm{T}, \mathrm{Xu} \mathrm{Y}$, et al. Survival patterns for patients with resected N2 non-small cell lung cancer and postoperative radiotherapy: a prognostic scoring model and heat map approach. J Thorac Oncol 2018;13:1968-74.

17. Chen B, Xia W, Wang Z, et al. Risk analyses of N2 lymphnode metastases in patients with T1 non-small cell lung cancer: a multi-center real-world observational study in China. J Cancer Res Clin Oncol 2019;145:2771-7.

18. Paz-Ares L, Spira A, Raben D, et al. Outcomes with durvalumab by tumour PD-L1 expression in unresectable, stage III non-small cell lung cancer in the PACIFIC trial. Ann Oncol 2020;31:798-806.

19. Forde PM, Chaft JE, Smith KN, et al. Neoadjuvant PD-1 Blockade in Resectable Lung Cancer. N Engl J Med 2018;378:1976-86.

20. Shu CA, Gainor JF, Awad MM, et al. Neoadjuvant atezolizumab and chemotherapy in patients with resectable non-small cell lung cancer: an open-label, multicentre, single-arm, phase 2 trial. Lancet Oncol 2020;21:786-95.

21. Uprety D, Mandrekar SJ, Wigle D, et al. Neoadjuvant immunotherapy for NSCLC: current concepts and future approaches. J Thorac Oncol 2020;15:1281-97.
Cite this article as: Zhang K, Cai J, Lu C, Zhu Q, Zhan C, Shen Y, Gu J, Ge D. Tumor size as a predictor for prognosis of patients with surgical IIIA-N2 non-small cell lung cancer after surgery. J Thorac Dis 2021;13(7):4114-4124. doi: 10.21037/jtd-21-428 